\section{Propofol as an adjunct during emergence from general anaesthesia}

To the Editor:

Propofol is useful not only as the sole agent for induction and maintenance of anaesthesia, but also as an adjunct during emergence after the use of a volatile anaesthetic. With the advent of procedures such as laparoscopy, it is often difficult to predict accurately when the procedure will end. Delivery of a volatile agent up to the end of the surgical procedure may delay emergence. In order to allow removal of the agent, its concentration is frequently reduced or eliminated in anticipation of completion of the case. Our timing is often misguided and the patient may require additional anaesthesia briefly during the final moments of surgery. Possible options include the use of: (1) an intravenous agent such as a barbiturate or propofol, (2) a narcotic, (3) a muscle relaxant, or (4) the volatile anaesthetic gas.

I have found that the use of a subanaesthetic dose of propofol, i.e., $10-30 \mathrm{mg}$ is sufficient to provide excellent anaesthesia at the final moments of surgery without delaying emergence. Due to its rapid distribution ${ }^{1-3}$ and metabolism, the use of propofol is a more logical choice than (1) a barbiturate which may delay emergence and produce a less rapidly oriented patient, (2) a narcotic which may produce prolonged respiratory depression, nausea and/or vomiting, (3) a muscle relaxant which if given in close proximity to the end of the procedure may make reversal difficult, or (4) the addition of a volatile agent which may reanaesthetize the patient.

Michael J. Peck MD

George Washington University Medical Center

Washington, DC 20037

\section{REFERENCES}

1 White $P F$. Propofol: pharmacokinetics and pharmacodynamics. Seminars in Anesthesia 1988; 7: 4-20.

2 Sebel PS, Lowdon JD. Propofol, a new intravenous anesthetic. Anaesthesia 1989; 71: 260-77.

3 Larijani GE, Gratz I, Afshar M, Jacobi AG. Clinical pharmacology of propofol: an intravenous anesthetic agent. Ann Pharmacol 1989; 23: 743-9.

\section{Gas mask during pregnancy and labour}

To the Editor:

The possible use of chemical weapons during the recent middle east war prompted the general use of gas masks by all civilians. Pregnant women, like the vast majority of the population, received a passive gas mask (without an electrical blower connected to the breathing apparatus).

We conducted a study to determine the influence of the passive gas mask on maternal oxygen saturation and fetal wellbeing by continous measurements of maternal pulse oximetry and fetal cardiotocographic monitoring respectively. Forty-eight nonsmoking pregnant women of $32-42$ wk gestation participated in the study. Twentyfour were in active labour with cervical dilatation of 3-7 $\mathrm{cm}$ and the remaining 24 were not in labour.

Simultaneous fetal heart rate monitoring and maternal pulse oximetry were performed $20 \mathrm{~min}$ before use of the gas mask, to serve as control measurements taken for 30 min after applying the mask. Gravidas with abnormal fetal heart rate pattern prior to the use of the gas mask were excluded from the study.

Maternal pulse oximetry did not decline while wearing the gas mask. The increased dead space imposed by the mask caused compensatory mild tachypnoea. No fetal heart rate abnormalities nor changes in the short and long-term variability were noted on fetal monitoring. The normal fetal cardiotocogram recorded while the gravida used the gas mask was a reliable indicator of fetal wellbeing. ${ }^{1}$ Apgar scores in all the newborns of patients wearing the gas mask in active labour were above 8 in $1^{\prime}$ and 9 in $10^{\prime}$.

Mean arterial oxygen saturation during pregnancy and delivery did not differ from that found in healthy nonpregnant patients. This was expected because a $10 \%$ increase in arterial oxygen partial pressure during pregnancy leads to virtually no change at the upper end of the oxyhaemoglobin dissociation curve. ${ }^{2}$ Nevertheless, although maternal $\mathrm{PO}_{2}$ was not measured, the normal $\mathrm{O}_{2}$ saturation combined with the normal fetal cardio-tocographic tracing suggest that wearing the passive gas mask when needed during pregnancy and delivery is safe.

Uriel Elchalal

Tiberiu Ezri*

David Soroker*

Avi Matzkel

Ariel Weissman

Department of Obstetrics and Gynecology, and

Department of Anaesthesiology*

Kaplan Hospital, Rehovot, affilated with the Hebrew

University Medical School and Hadassah Hospital Jerusalem, Israel 